Paediatr. Paedolog. 2020 · 55:306-313 https://doi.org/10.1007/s00608-020-00848-4 Online publiziert: 24 . November 2020 (c) Der/die Autor(en) 2020

\section{Anja Christina WeinhandI ${ }^{1,2}$. Winfried RebhandI ${ }^{1,2}$}

' Universitätsklinik für Kinder- und Jugendchirurgie, Medizinische Universität Wien, Wien, Österreich

${ }^{2}$ Klinische Abteilung für Kinderchirurgie, Medizinische Universität Wien, Wien, Österreich

\title{
Therapie der angeborenen Thoraxfehlbildungen - Rückblick und Ausblick
}

Die Trichterbrust (Pectus excavatum) und die Kielbrust (Pectus carinatum) zählen mit einer Inzidenz von etwa 0,1 bis 0,8 pro 100 Neugeborene $\mathrm{zu}$ den häufigsten angeborenen Thoraxwandfehlbildungen. Vorwiegend sind Jungen betroffen; das Geschlechterverhältnis liegt zwischen 2:1 und 9:1 männlich zu weiblich. Mit $90 \%$ tritt die Trichterbrust am häufigsten auf. Kennzeichnendes Merkmal für diese Fehlbildung ist die Einsenkung des Brustbeins. Kielbrustfehlbildungen und diverse Mischformen kommen deutlich seltener vor.

Die Ursache der angeborenen Thoraxdeformitäten ist nach wie vor nicht vollständig geklärt. Mehrheitlich diskutiert wird ein abnormales Wachstum der chondrokostalen Rippenknorpel, das da$\mathrm{zu}$ führt, dass das Brustbein entweder nach innen (Trichterbrust) oder nach außen (Kielbrust) gedrückt wird (• Abb. 1). Auch eine familiäre Häufung von Brustwanddeformitäten ist beschrieben, wobei der exakte Vererbungsmodus ebenfalls noch unbekannt ist. Des Weiteren besteht die Assoziation zu anderen Erkrankungen bzw. Fehlbildungen wie der Skoliose, dem Marfan- oder Poland-Syndrom.

Die Diagnosestellung erfolgt in der Regel bei der Geburt oder innerhalb der ersten Lebensjahre, in denen die Ausprägung der Deformität meist noch moderat ist. Mit fortschreitendem Wachstum, besonders während der Pubertät, ist die Befundprogredienz häufig besonders markant und es können erstmalig damit assoziierte Beschwerden auftreten. Das

Literaturnachweise beim Verfasser klinische Beschwerdebild ist gewöhnlich heterogen (•Tab. 1) und korreliert mit dem Ausprägungsgrad der Fehlbildung. Langfristig kann eine Einschränkung der kardiopulmonalen Leistungsfähigkeit resultieren, die häufig die Indikation zur operativen Korrektur stellt. Neben den vornehmlich körperlichen Einschränkungen leiden die Betroffenen aber auch oft unter dem kosmetischen Aspekt der Brustkorbfehlbildung. Vor allem die Befundprogredienz in der Pubertät kombiniert mit dem gesellschaftlichen und medialen Schönheitsdruck ist für viele Patient und Patientinnen sehr belastend. Somit ist auch die psychosoziale Komponente der Thoraxdeformitäten nicht ganz außer Acht zu lassen.

\section{Anamnese, klinische Unter- suchung und Zusatzdiagnostik}

Bei der klinischen Untersuchung werden zuerst Art und Ausprägung der Deformität evaluiert und fotodokumentiert. Dies ist Grundvoraussetzung vor jeglichem Behandlungsbeginn, um $\mathrm{zu}$ ermitteln, von welchem Behandlungskonzept die Patientinnen und Patienten am meisten profitieren. Ergänzend dazu werden weiterführend spezifische $\mathrm{Zu}$ satzuntersuchungen veranlasst und nach assoziierten Fehlbildungen gesucht. Hier vor allem nach phänotypischen Zeichen für einen Morbus Marfan und begleitenden orthopädischen Problemen, z. B. einer Skoliose.

Die Bildgebung nimmt in der Zusatzdiagnostik den größten Stellenwert ein. Das native Thoraxröntgen wurde von den sehr viel spezifischeren Schnittbildunter- suchungen wie der Computertomographie (CT) und der Magnetresonanztomographie (MRT) abgelöst. Diese Untersuchungen geben nicht nur Auskunft über die Anatomie des Thorax, die Einbeziehung kardiopulmonaler Strukturen sowie den umgebenden Weichteilmantel, sondern lassen auch gleich die exakte Ausprägung der Deformität bestimmen und objektivieren (• Abb. 2 und 3). Hierfür hat sich bei der Trichterbrust der Haller-Index etabliert (• Abb. 3). Im CT-Querschnittbild wird auf Höhe des tiefsten Punkts des Trichters der Quotient aus intrathorakalem Querdurchmesser und anteroposteriorem Abstand zwischen Sternumhinterkante und Wirbelkörpervorderkante berechnet. Bei Werten zwischen 2,5 und 2,7 wird von einem Normalbefund gesprochen; liegt der Haller-Index bei 3,2 oder höher, wird häufig die Empfehlung für eine Operation ausgesprochen.

Welche der beiden Untersuchungsmodalitäten veranlasst wird, obliegt dem Behandler und sollte individuell

Tab. 1 Klinisches Beschwerdebild

Mögliche assoziierte Symptome

Thorakale Kyphose

Belastungsdyspnoe

Eingeschränkte körperliche Belastbarkeitskapazität

Thoraxschmerzen

Tachykardie und Palpationen

Soziale Isolation durch Ästhetikproblem

Asthmatische Beschwerden

Schwindel und erhöhte Kollapsneigung

Gastroösophageale Refluxbeschwerden 

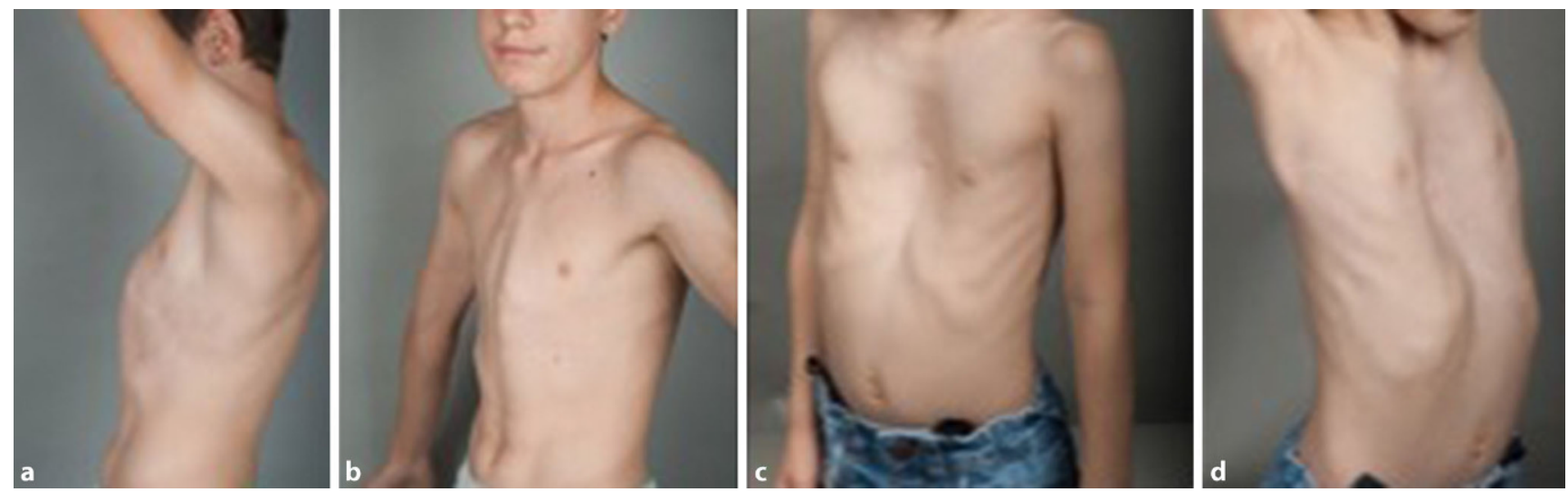

Abb. 1 \ Patient mit Kielbrust (a, b) und Patient mit Trichterbrust (c, d; Bilder aus dem eigenen Archiv; Universitätsklinik für Chirurgie, Abteilung für Kinder- und Jugendchirurgie, Medizinische Universität Wien)

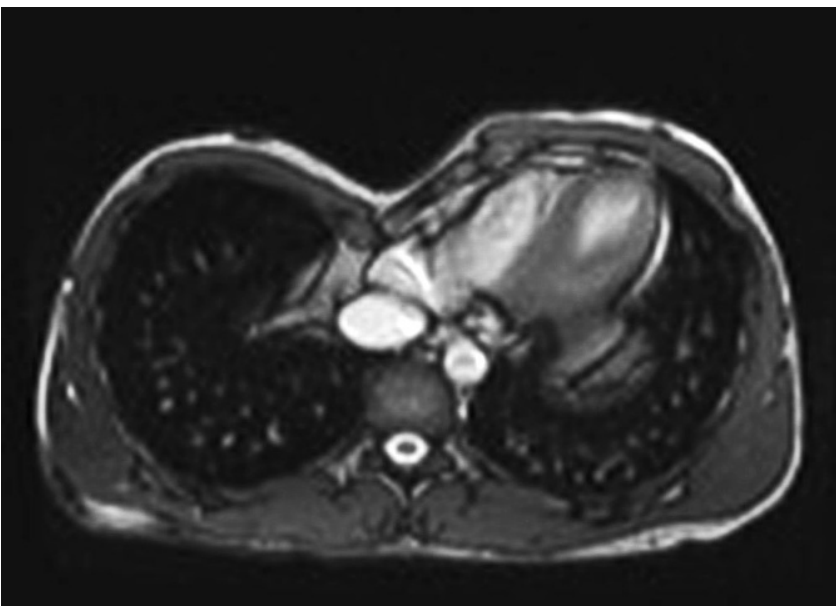

Abb. $2 \triangleleft$ Magnetresonanztomogramm eines Trichterbrustpatienten (Bilder aus dem eigenen Archiv; Universitätsklinik für Chirurgie, Abteilung für Kinder-und Jugendchirurgie und Universitätsklinik für Radiologie; Medizinische Universität Wien)

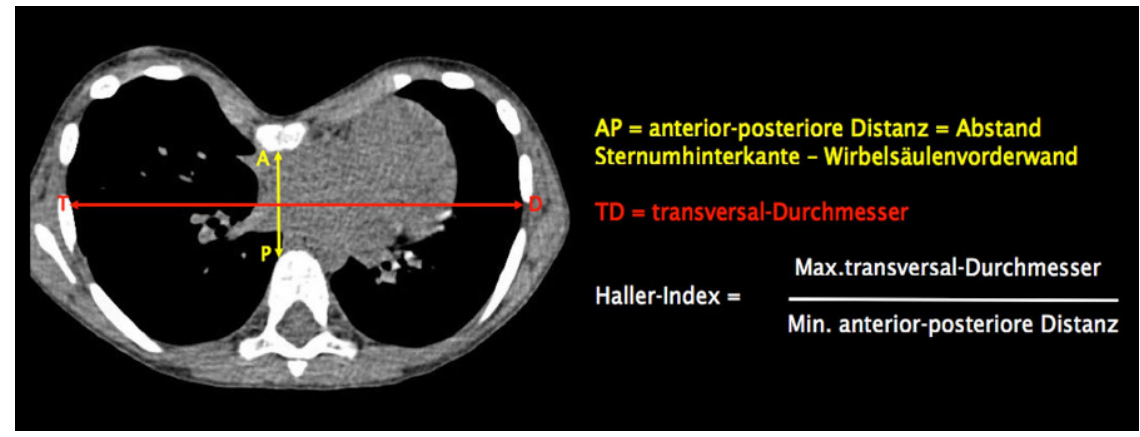

Abb. $3 \Delta$ Computertomogramm eines Trichterbrustpatienten mit Berechnung des Haller-Index (Bilder aus dem eigenen Archiv; Universitätsklinik für Chirurgie, Abteilung für Kinder- und Jugendchirurgie und Universitätsklinik für Radiologie; Medizinische Universität Wien)

gewählt werden. $\mathrm{Zu}$ beachten ist, dass sich das ausgewählte Verfahren meist in der Durchführung sowie in den angewandten Untersuchungsprotokollen erheblich von der Routinediagnostik unterscheidet. So ist vor allem bei einer bevorstehenden Operation das Befunden durch mit diesen Fehlbildungen vertrau- ven generell dennoch groß genug sind, sind die meisten Patientinnen und $\mathrm{Pa}$ tienten zuerst durch das eingeschränkte Auswurfvolumen des Herzens limitiert.

Thoraxfehlbildungen können symmetrisch oder asymmetrisch ausgeprägt sein. Bei einer asymmetrischen Trichterbrust ist das Sternum zur tieferen Seite hin rotiert (• Abb. 3). Bei der Kielbrust besteht meistens eine symmetrische Protrusion des Sternums. Aber auch hier kommen asymmetrische Varianten und unterschiedliche Typen vor. Eine sehr übersichtliche Gliederung dazu stammt von Yuksel et al. (• Abb. 4). Additiv zur Bildgebung kommt bei der Abklärung der Kielbrust auch ein sogenannter Sternum-Kompressionstest zum Einsatz, um die Flexibilität des Brustbeins $\mathrm{zu}$ determinieren, da diese ausschlaggebend für die Auswahl der weiteren Therapie ist. Bei guter Flexibilität des Sternums ist eine konservative Therapie mit entsprechenden Kompressionsorthesen möglich, während bei rigiderem Sternum primär die operative Korrektur indiziert ist. Das ausgewählte operative Verfahren ist neben der Variante der vorliegenden Kielbrust ebenfalls abhängig von der Sternumflexibilität. So ist beispielweise bei hohen Sternumdrücken meist eine minimal-invasive Operation möglich, während bei Kielbrustformen mit starker Knickbildung des Sternums (Pectus arcuatum; - Abb. 4b) ein offenes Vorgehen (OP nach Ravitch) indiziert ist. 


\section{Therapie}

Es existiert mittlerweile ein breites Spektrum an konservativen und chirurgischen Behandlungskonzepten in der Therapie angeborener Thoraxfehlbildungen. Das Entscheidendste ist immer, die ausgewählte Therapie individuell an das Beschwerdebild der Patienten und Patientinnen und die jeweils vorliegende Thoraxdeformität anzupassen.

\section{Konservative Therapieansätze}

Der nichtoperativen Behandlung sollte in der Behandlung von Brustkorbdeformitäten stets der Vorzug gegeben werden, vor allem bei jungen Patientinnen und Patienten, denn je jünger diese sind, desto höher liegen die Erfolgschancen. Mittlerweile existieren in diesem Bereich verschiedenste Therapiekonzepte. Die Anwendung orthopädisch angepasster Kompressionsorthesen sowie die Vakuumsaugglockentherapie haben sich hierbei am häufigsten durchgesetzt.

\section{Physiotherapeutische Maßnahmen}

Gerade geringgradig ausgebildete Brustkorbdeformitäten lassen sich durch gezielte physiotherapeutische Übungen in Kombination mit Muskeltraining gut verbessern und führen überdies hinaus bei den Betroffenen auch zu einer positiveren Körperwahrnehmung und Steigerung des Selbstwertgefühls. Auch als vorbereitende Maßnahmen vor einer geplanten Operation können sie unterstützend sein. Die entsprechenden Übungen werden gemeinsam mit spezialisierten Physiotherapeutinnen und -therapeuten erlernt und dann im Idealfall regelmäßig selbständig fortgesetzt. Bei zusätzlichen Deformierungen oder Erkrankungen der Wirbelsäule empfiehlt sich auch eine fachärztliche orthopädische Begutachtung.

\section{Vakuumtherapie mittels Saugglocke}

Die Vakuumsaugglockentherapie (nach Eckart Klobe; Abb. 5) bei der Trichterbrust hat das Ziel, durch regelmäßige Anwendung eine permanente Anhebung des Sternums sowie der sternokostalen

Paediatr. Paedolog. 2020 · 55:306-313 https://doi.org/10.1007/s00608-020-00848-4

(c) Der/die Autor(en) 2020

\section{A. C. Weinhandl $\cdot$ W. Rebhandl}

\section{Therapie der angeborenen Thoraxfehlbildungen - Rückblick und Ausblick}

\section{Zusammenfassung}

Thoraxfehlbildungen umfassen ein breites Spektrum angeborener Fehlbildungen mit unterschiedlichster phänotypischer und klinischer Manifestation. Diagnostik und Betreuung der Patientinnen und Patienten erfordern viel Erfahrung und Expertise sowie die Anbindung an ein multidisziplinäres Behandlungsteam. Die operative Korrektur erfolgt meist im Kindes- und Jugendalter und ist daher seit Jahrzehnten eine Domäne der Kinder- und Jugendchirurgie.

Eine operative Korrektur ist nur dann sinnvoll, wenn dadurch eine wesentliche Besserung der Symptomatik erzielt werden kann. Bei weniger schwerwiegenden Deformitäten oder auch in Grenzfällen ist zunächst ein konservatives Vorgehen bestehend aus gezielter Physiotherapie und Anwendung entsprechender Orthesen ratsam. Bei Ausbleiben des gewünschten Therapieerfolgs oder wenn die Betroffenen einer konservativen Therapie gänzlich ablehnend gegenüberstehen, ist die nächste Behandlungsoption die Operation. Hier haben sich hauptsächlich die minimalinvasiven Vorgehensweisen durchgesetzt, da diese mit kürzerer Operationsdauer, früherer Mobilisierung, besserem und stabilerem postoperativen Ergebnis und geringerem Narbenbildungsrisiko einhergehen. Zusammenfassend lässt sich also festhalten, dass zahlreiche an den jeweiligen Schweregrad der Deformierung angepasste Therapiekonzepte für angeborene Thoraxdeformitäten existieren. Sobald ein Wunsch nach Behandlung besteht bzw. körperliche Beschwerden in Erscheinung treten, ist die Anbindung jener Patientinnen und Patienten an ein Spezialzentrum mit auf diese Fehlbildungen spezialisierten Kinderund Jugendchirurgen und -chirurginnen empfohlen. Dort können diese Patientinnen und Patienten entsprechend den neuesten wissenschaftlichen und operativen Standards und größtmöglicher fachlicher Expertise betreut werden.

Schlüsselwörter

Trichterbrust · Kielbrust · Kinderchirurgie · Therapie · Diagnostik

\section{Congenital chest wall deformities-state of play and where to go}

\section{Abstract}

Congenital chest wall deformities represent a wide spectrum of anomalies of chest wall growth leading to an altered structure and anatomy of the sternum, the rib cage, the ribs itself and the spine. In severe cases these alterations could lead to significant physical and/or cardiopulmonary symptoms, exercise limitations, and aesthetic concerns. Depending on the patient's age, the severity of the deformity and the associated symptoms treatment becomes necessary. Close observation of the deformity during the growth period allows the pediatric surgeon to present various treatment strategies to the patient and parents. Nonsurgical treatment, such as orthotic bracing or the use of the vacuum bell, combined with physical therapy, should be considered as the treatment of choice. This approach has shown to be effective in the majority of patients, especially in pediatric and young adolescent patients. If these conservative treatment strategies fail, considerations about surgical correction would be the next step. Surgical repair of congenital chest wall deformities requires a multidisciplinary exspertise particulary when it comes to the pediatric surgeon's competence. To guarantee a maximal functional and aesthetic outcome, timing of treatment should be carefully anticipated.

\section{Keywords}

Funnel chest · Keel chest · Pediatric surgery · Therapy · Diagnostic
Übergänge zu bewirken und dadurch den Trichter auszukorrigieren. Die individuelle Behandlungsdauer ist dabei abhängig von der Thoraxelastizität und der Trichtertiefe, dem Patient/innenalter und entscheidend von der Tragecompliance. Eine detaillierte Beschreibung sowie Hinweise zur genauen Anwendung finden sich in dem von E. Klobe herausgegebenen Anwendermanual. 
Hier steht eine Anzeige.

\section{曾 Springer}



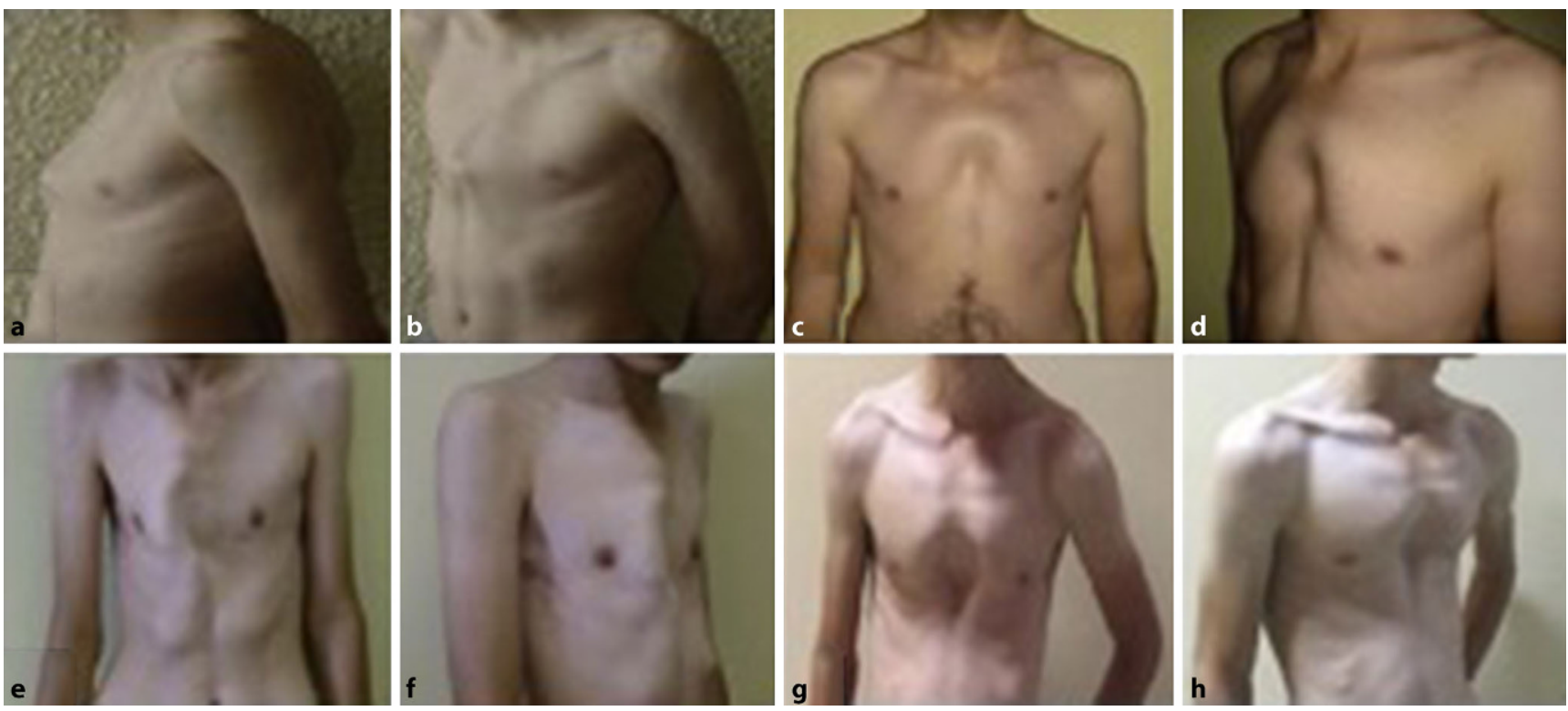

Abb. $4 \Delta$ Verschiedene Kielbrustvarianten (mit freundlicher Genehmigung von Prof. Mustafa Yuksel, Marmara Universität Istanbul, Türkei). Klassische Kielbrust (a, b); chrondromanubriale Kielbrustvariante oder Pectus arcuatum (c, d); asymmetrische Kielbrust $(\mathbf{e}, \mathbf{f})$; ,Horse Shoe-Chest" $(\mathbf{g}, \mathbf{h})$
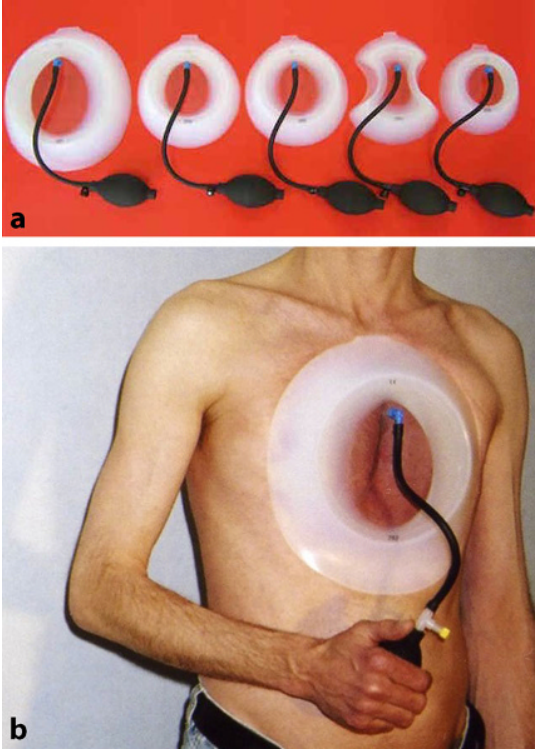

Abb. $5 \Delta$ a Die 5 verschiedenen Saugglockentypen nach EckartKlobe: große Saugglocke $(26 \mathrm{~cm})$ für männliche Personen mit einer Körpergröße von mehr als ca. 1,70 m; kleine Saugglocke $(19 \mathrm{~cm})$ für (männliche) Personen mit einer Körpergröße von mehr als ca. 1,40 m; kleine Saugglocke Typ „Bodybuilder" $(19 \mathrm{~cm})$ für Bodybuilder und spezielle Anwendungen; kleine Saugglocke Typ "Frauen" $(19 \mathrm{~cm})$ für Frauen und Mädchen ab Beginn der Brustentwicklung; Mini-Saugglocke $(16 \mathrm{~cm})$ für Kinder mit einer Körpergröße von mehr als ca. 1,05 m. b Anwendung am Patienten (Bild mit freundlicher Genehmigung von Eckart Klobe; $\odot$ Eckart Klobe)

\section{Kompressionsorthesen mittels Pelotte}

Auch bei der Kielbrust haben sich nichtinvasive Behandlungsmethoden als fixer Bestandteil in der konservativen Therapie etabliert. In erster Linie kommt hierbei die Kompressionsorthesentherapie mittels Pelotte (• Abb. 6) zum Einsatz. Das Wirkprinzip besteht in einer Druckausübung von außen auf das Brustbein, wodurch dieses entsprechend remodelliert werden soll. Der Behandlungserfolg hängt neben der Sternumflexibilität von den gleichen Faktoren ab wie bei der Saugglockenanwendung.

\section{Chirurgische Therapieansätze}

Bei erfolgloser konservativer Behandlung oder Ablehnung dieser durch die Betroffenen ist als nächste Therapieoption die operative Korrektur kritisch zu diskutieren. In den vergangenen Jahrzehnten haben sich viele unterschiedliche Operationstechniken zur Korrektur angeborener Brustkorbdeformitäten entwickelt. Die früher hauptsächlich angewandten offenen Korrekturverfahren (Ravitch, Rehbein, diverse Modifikationen) sind inzwischen großteils von den minimal-invasiven Verfahren (MIRPE, OP nach Nuss) abgelöst worden. Diese gehen mit einem postoperativ stabi- leren Ergebnis sowie einer verkürzten Operations- und stationären Aufenthaltsdauer einher. Ebenso wird neben der bestmöglichen Korrektur der Brustkorbfehlbildung auch ein geringeres Narbenbildungsrisiko berücksichtigt.

\section{Operative Korrektur der Trichterbrust (Pectus excavatum)}

Bei der Operation nach Ravitch-Rehbein werden das Sternum - teils via Sternumosteotomie - und die chondrokostalen Knorpelübergänge dargestellt und die deformierten Rippenknorpel unter Belassung des Perichondriums reseziert. Anschließend erfolgt die Rekonstruktion der vorderen Thoraxwand mit unterschiedlichen Osteosynthesematerialien (z. B. Metallbügel). Die Korrekturergebnisse sind in der Regel zufriedenstellend, jedoch nicht vergleichbar mit den Ergebnissen der Nuss-OP, insbesondere in Bezug auf die postoperative Stabilität. Ebenfalls geht diese sehr invasive Technik mit großen Wundflächen, entsprechend langen OP-Narben und erheblichen postoperativen Schmerzen einher, weswegen sie inzwischen mehrheitlich zugunsten der minimal-invasiven Korrektur nach Donald Nuss (MIRPE) verlassen wurde. Bei dieser Operation wird unter thorakoskopischer Kontrolle über zwei kleine, in der vorderen Axillarlinie verlau- 
fende Hautschnitte ein Metallbügel unter das Sternum implantiert (• Abb.7a). Dieser verbleibt im Normalfall 2-3 Jahre in situ, um eine dauerhafte Stabilisierung des Operationsergebnisses zu erreichen. Häufigste Komplikation dieses Eingriffs ist der Pneumothorax, gefolgt vom Hämatothorax sowie einer Überkorrektur der Deformität. Auch Dislokationen des Bügels oder der Stabilisatorplatten können vorkommen. In der jüngsten Vergangenheit haben sich zahlreiche Modifikationen dieser Operation entwickelt, insbesondere in Bezug auf Länge und Anzahl der implantierten Bügel. Eine der momentan neuesten Techniken auf diesem Gebiet stammt von Prof. Mustafa Yuksel (Marmara Universität Istanbul, Türkei). Er verwendet je nach vorliegender Deformität kürzere Bügel (Shortbar-Methode) oder auch mehrere Bügel, die nicht nur horizontal, sondern auch schräg oder gekreuzt positioniert werden können (Cross-bar- oder Double-humpMethode; • Abb. 8). Insbesondere bei äl- teren Patientinnen und Patienten mit rigiderem Brustkorb; bei stark ausgeprägten Deformitäten oder asymmetrischen Formen erweist sich dies als enormer Vorteil. Durch die ventrale Fixation der Stabilisatorplatte an der Thoraxwand ist der Bügel zudem deutlich rotationsstabiler (• Abb. 7b) und kann kaum mehr abkippen.

\section{Operative Korrektur der Kielbrust (Pectus carinatum)}

Auch die Kielbrust wird heutzutage nach Möglichkeit vornehmlich minimal-invasiv operiert. Analog zur Nuss-OP wird bei der minimal-invasiven Kielbrustkorrektur (MIRPC) ein Metallbügel verwendet, um die Deformität auszukorrigieren. Dieser wird ebenfalls über kleine seitliche Hautschnitte extrathorakal-submuskulär vor das Brustbein gesetzt. Daher wird dieser Eingriff gern auch als inverses Nuss-Procedere bezeichnet. Der individuell geformte Bügel wird dann in seitlich auf den Rippen fixierte Sta- bilisatoren eingespannt und drückt dadurch das Brustbein nach unten. Bei einer Pectus arcuatum ist ein minimal-invasives Vorgehen hingegen kontraindiziert. Hier kommt der modifizierte Ravitch zum Einsatz: Über einen quer geführten Hautschnitt wird durch Elevation der Brustmuskulatur die Sternumdeformität freigelegt und die entsprechend verformten Rippenknorpel werden reseziert. Nach der Sternumosteotomie werden Brustbein und Rippen in die gewünschte Stellung gebracht und fixiert.

\section{Postoperativer Verlauf und Schmerztherapie}

Postoperativ sind eine adäquate Analgesie und rasche Mobilisation der Patienten und Patientinnen von entscheidender Bedeutung im Genesungsprozess. Zur suffizienten Schmerztherapie stehen regionalanästhesiologische Verfahren (z.B. Peri-/Epiduralkatheter, PainBuster $^{\circledR}$, B. Braun Melsungen AG | Hospital Care, Melsungen, Deutschland)

Hier steht eine Anzeige. 

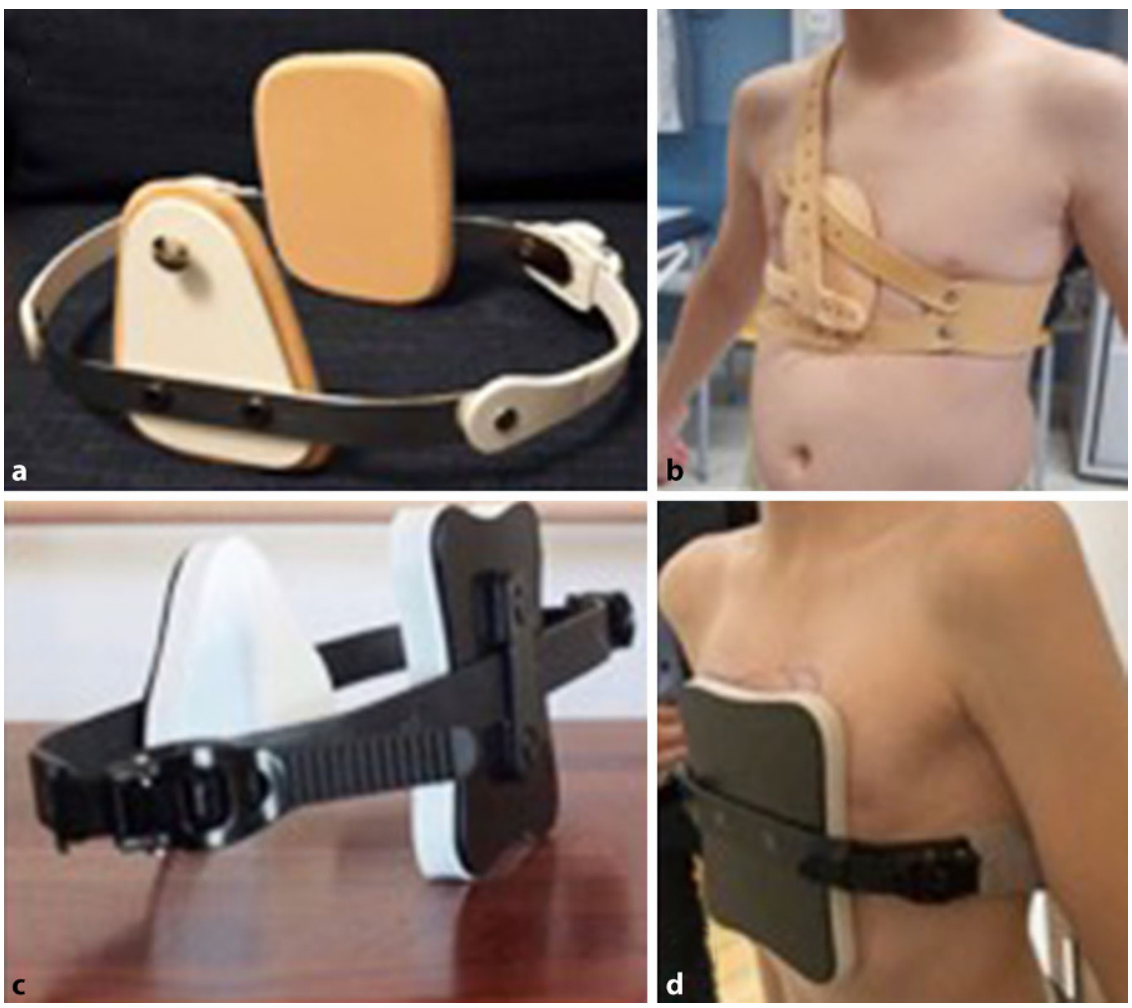

Abb. 6 ॥ Verschiedene Kompressionsorthesenmodelle. a Pelotte nach "Wiener Art“; b-d Pelottenanwendung am Patienten ( $\mathbf{a}, \mathbf{b}$ Bilder aus dem eigenen Archiv; Universitätsklinik für Chirurgie, Abteilung für Kinder- und Jugendchirurgie, Medizinische Universität Wien; $\mathbf{c}, \mathbf{d}$ Bild mit freundlicher Genehmigung von Prof. Mustafa Yuksel, Marmara Universität Istanbul, Türkei)
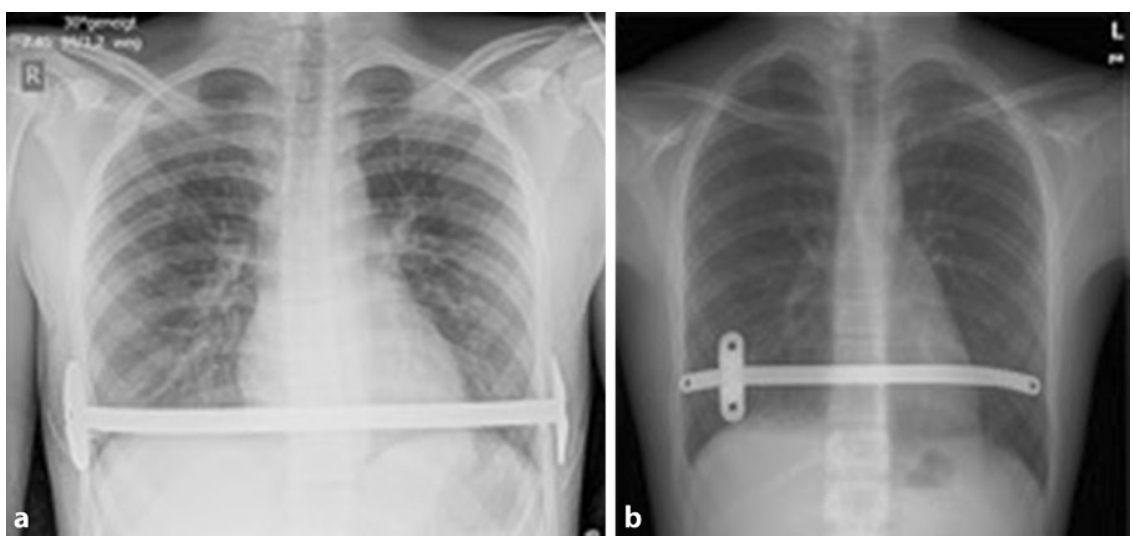

Abb. $7 \triangle$ OP nach Nuss (a) und modifizierter Variante nach M. Yuksel mit ventraler Fixation der Stabilisatorplatte (b) (Bilder aus dem eigenen Archiv; Universitätsklinik für Chirurgie, Abteilung für Kinderund Jugendchirurgie und Universitätsklinik für Radiologie; Medizinische Universität Wien)

sowie intravenöse und orale Analgetika (entsprechend dem WHO-Stufenschema) zur Verfügung. Als Infektionsprophylaxe wird üblicherweise über die ersten $72 \mathrm{~h}$ postoperativ eine parenterale Antibiose verabreicht. Sport, ausgenommen Kontaktsport, kann nach einem Monat postoperativ in moderatem Aus- maß und nach 3 Monaten wieder ohne Einschränkung ausgeübt werden.

\section{Zusammenfassung}

Thoraxfehlbildungen umfassen ein breites Spektrum angeborener Fehlbildungen mit unterschiedlichster phänotypischer und klinischer Manifestation. Dia- gnostik und Betreuung der Patientinnen und Patienten erfordern viel Erfahrung und Expertise sowie die Anbindung an ein multidisziplinäres Behandlungsteam. Die operative Korrektur erfolgt meist im Kindes- und Jugendalter und ist daher seit Jahrzehnten eine Domäne der Kinder- und Jugendchirurgie.

Eine operative Korrektur ist nur dann sinnvoll, wenn dadurch eine wesentliche Besserung der Symptomatik erzielt werden kann. Bei weniger schwerwiegenden Deformitäten oder auch in Grenzfällen ist zunächst ein konservatives Vorgehen bestehend aus gezielter Physiotherapie und Anwendung entsprechender Orthesen ratsam. Bei Ausbleiben des gewünschten Therapieerfolgs oder wenn die Betroffenen einer konservativen Therapie gänzlich ablehnend gegenüberstehen, ist die nächste Behandlungsoption die Operation. Hier haben sich hauptsächlich die minimal-invasiven Vorgehensweisen durchgesetzt, da diese mit kürzerer Operationsdauer, früherer Mobilisierung, besserem und stabilerem postoperativen Ergebnis und geringerem Narbenbildungsrisiko einhergehen.

Zusammenfassend lässt sich also festhalten, dass zahlreiche an den jeweiligen Schweregrad der Deformierung angepasste Therapiekonzepte für angeborene Thoraxdeformitäten existieren. Sobald ein Wunsch nach Behandlung besteht bzw. körperliche Beschwerden in Erscheinung treten, ist die Anbindung jener Patientinnen und Patienten an ein Spezialzentrum mit auf diese Fehlbildungen spezialisierten Kinder- und Jugendchirurgen und -chirurginnen empfohlen. Dort können diese Patientinnen und Patienten entsprechend den neuesten wissenschaftlichen und operativen Standards und größtmöglicher fachlicher Expertise betreut werden.

\section{Conclusion}

Congenital chest wall deformities represent a wide spectrum of anomalies of chest wall growth leading to an altered structure and anatomy of the sternum, the rib cage, the ribs itself and the spine. In severe cases these alterations could lead to significant physical and/or cardiopulmonary symptoms, exercise limitations, 

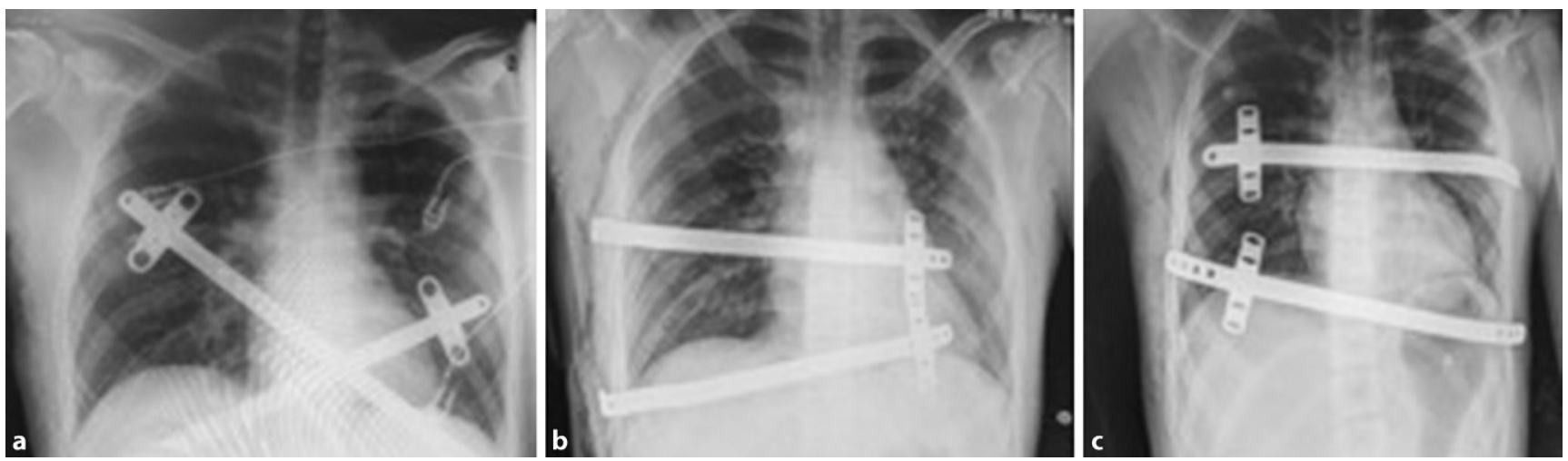

Abb. 8 A Cross-bar- und Double-hump-Methode nach M. Yuksel (Bilder aus dem eigenen Archiv; Universitätsklinik für Chirurgie, Abteilung für Kinder- und Jugendchirurgie und Universitätsklinik für Radiologie; Medizinische Universität Wien)

and aesthetic concerns. Depending on the patient's age, the severity of the deformity and the associated symptoms treatment becomes necessary. Close observation of the deformity during the growth period allows the pediatric surgeon to present various treatment strategies to the patient and parents. Nonsurgical treatment, such as orthotic bracing or the use of the vacuum bell, combined with physical therapy, should be considered as the treatment of choice. This approach has shown to be effective in the majority of patients, especially in pediatric and young adolescent patients. If these conservative treatment strategies fail, considerations about surgical correction would be the next step. Surgical repair of congenital chest wall deformities requires a multidisciplinary exspertise particulary when it comes to the pediatric surgeon's competence. To guarantee a maximal functional and aesthetic outcome, timing of treatment should be carefully anticipated.

\section{Take Home Message}

- Trichter- und Kielbrustfehlbildungen zählen zu den häufigsten angeborenen Malformationen der vorderen Thoraxwand.

- Das klinische Beschwerdebild ist stets heterogen.

- Behandlung und Therapie erfolgen sowohl konservativ als auch operativ.

- Idealer Versorgungszeitpunkt ist die frühe Adoleszenz.

- Die optimale Betreuung erfolgt in einem Zentrum mit darauf spezialisier- ten Kinder- und Jugendchirurginnen und -chirurgen.

\begin{tabular}{ll}
\hline Korrespondenzadresse \\
Univ.-Prof. Univ.-Doz. Dr. \\
med. Winfried RebhandI \\
Universitätsklinik für Kinder- \\
und Jugendchirurgie, \\
Medizinische Universität \\
Wien \\
Wien, Österreich \\
क Katharina \\
Steinbrecher \\
Fotografie
\end{tabular}

Funding. Open access funding provided by Medical University of Vienna.

\section{Einhaltung ethischer Richtlinien}

Interessenkonflikt. A.C. Weinhandl und W. Rebhandl geben an, dass kein Interessenkonflikt besteht.

Für diesen Beitrag wurden von den Autoren keine Studien an Menschen oder Tieren durchgeführt. Für die aufgeführten Studien gelten die jeweils dort angegebenen ethischen Richtlinien.

Open Access. Dieser Artikel wird unter der Creative Commons Namensnennung 4.0 International Lizenz veröffentlicht, welche die Nutzung, Vervielfältigung, Bearbeitung, Verbreitung und Wiedergabe in jeglichem Medium und Format erlaubt, sofern Sie den/die ursprünglichen Autor(en) und die Quelle ordnungsgemäß nennen, einen Link zur Creative Commons Lizenz beifügen und angeben, ob Änderungen vorgenommen wurden.

Die in diesem Artikel enthaltenen Bilder und sonstiges Drittmaterial unterliegen ebenfalls der genannten Creative Commons Lizenz, sofern sich aus der Abbildungslegende nichts anderes ergibt. Sofern das betreffende Material nicht unter der genannten Creative Commons Lizenz steht und die betreffende Handlung nicht nach gesetzlichen Vorschriften erlaubt ist, ist für die oben aufgeführten Weiterverwendungen des Materials die Einwilligung des jeweiligen Rechteinhabers einzuholen.

Weitere Details zur Lizenz entnehmen Sie bitte der Lizenzinformation auf http://creativecommons.org/ licenses/by/4.0/deed.de.

Hinweis des Verlags. Der Verlag bleibt in Hinblick auf geografische Zuordnungen und Gebietsbezeichnungen in veröffentlichten Karten und Institutsadressen neutral. 